\title{
Evaluation of the Degradation on a COTS Linear CCD Induced by Total Ionizing Dose Radiation Damage
}

\author{
Zujun Wang, Baoping He, Wuying Ma, Zhibin Yao, Shaoyan Huang, \\ Minbo Liu, and Jiangkun Sheng
}

State Key Laboratory of Intense Pulsed Irradiation Simulation and Effect, Northwest Institute of Nuclear Technology, P.O. Box 69-10, Xian 710024, China

Correspondence should be addressed to Zujun Wang; wzj029@qq.com

Received 16 April 2016; Revised 22 June 2016; Accepted 27 June 2016

Academic Editor: Mengmeng Wang

Copyright (C) 2016 Zujun Wang et al. This is an open access article distributed under the Creative Commons Attribution License, which permits unrestricted use, distribution, and reproduction in any medium, provided the original work is properly cited.

\begin{abstract}
The evaluation of the degradation on a COTS linear Charge Coupled Device (CCD) induced by total ionizing dose (TID) radiation damage was presented. The radiation experiments were carried out at a ${ }^{60} \mathrm{Co} \gamma$-ray source. The parameters of DALSA's linear CCD were measured at the CCD test systems as the EMVA1288 standard before and after the radiation. The dark current, dark signal nonuniformity (DSNU), photo response nonuniformity (PRNU), saturation output, full-well capacity (FWC), quantum efficiency $(\mathrm{QE})$, and responsivity versus the TID were analyzed. The behavior of the tested CCD had shown a remarkable degradation after radiation. The degradation mechanisms of the CCD induced by TID damage were also discussed.
\end{abstract}

\section{Introduction}

Charge Coupled Device (CCD) has been extensively used in imaging, signal processing, and serial memories, especially for particle detection and space applications $[1,2]$. The CCDs operated in space environments are very vulnerable to space radiation damage. In general, two types of CCD radiation damage are referred to as ionizing and displacement damage [3]. ${ }^{60}$ Co $\gamma$-ray radiation mainly causes ionizing damage. Ionizing damage can cause the oxide traps and interface traps increase in the CCDs. This damage may induce the obvious degradations of CCD parameters such as the increase of dark current, dark signal nonuniformity (DSNU), and photo response nonuniformity (PRNU) and the decrease of saturation output, full-well capacity (FWC), quantum efficiency $(\mathrm{QE})$, responsivity. The increase of dark current caused by radiation induces the decrease of the effective signal and the imaging quality. When the dark currents (dark signals) are full of the potential wells, the CCD will be functional failure. The increase of the DSNU and the PRNU caused by radiation induces the increase of the inhomogeneities of pixel output, which decreases the imaging uniformity. The decrease of saturation output and FWC caused by radiation induces the decrease of imaging quality such as the dynamic range
(DR) and the signal-to-noise ratio (SNR). The decrease of the $\mathrm{QE}$ and responsivity caused by radiation induces the decrease of the photon-sensitivity of a CCD. These parameters are well known as the radiation sensitive parameters of a CCD. The degradations of these parameters can severely affect the performance of a CCD.

Simone et al. have presented the dark current density and threshold shift of hardening CCDs versus the accumulated dose and annealing time [4]. Hopkinson has investigated the methods which can restrain the increase of dark current in the CCDs induced by total ionizing dose (TID) [5]. Wang et al. have studied the degradation of the saturation output signal voltage of the array CCDs caused by the TID radiation damage [6]. However, fewer papers laid emphasis on the TIDinduced degradations of QE, responsivity, PRNU, and FWC in the CCDs. DALSA's linear CCDs have been widely used for space applications in recent years. Few papers focus on the radiation effects on this type of CCD. The evaluation of the degradations on DALSA's linear CCDs induced by total ionizing dose (TID) damage is important to the satellite designers.

The research reported herein examines the experiments of TID radiation effects on DALSA's linear CCDs. The degradations of the parameters induced by TID damage were evaluated. The dark current, DSNU, PRNU, saturation output, 


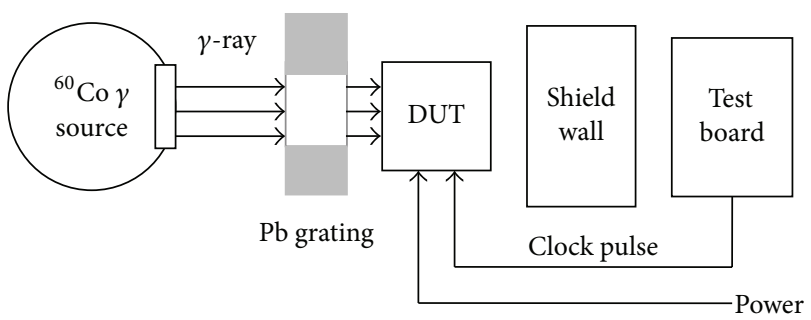

FIGURE 1: Experimental setup for a CCD TID radiation test.

and FWC as a function of TID at $30.0 \mathrm{rad}(\mathrm{Si}) / \mathrm{s}$ were presented. The $\mathrm{QE}$ and responsivity as a function of wavelength before and after $20 \mathrm{krad}(\mathrm{Si})$ radiation were also presented. The degradation mechanisms of the CCD induced by TID damage were analyzed in detail.

\section{Experimental Details}

The radiation experiments were carried out at a ${ }^{60} \mathrm{Co} \gamma$-ray source. The CCD was unbiased with all pins grounded during ${ }^{60} \mathrm{Co} \gamma$ radiation and tested at $30.0 \mathrm{rad}(\mathrm{Si}) / \mathrm{s}$. The $\mathrm{CCD}$ was measured at the TID of 5, 10, 15, and $20 \mathrm{krad}(\mathrm{Si})$. The CCD parameters were tested within $30 \mathrm{~min}$ after each radiation step. The output video signals were tested with illumination and dark conditions. The parameters were measured before and after radiation at the CCD test equipment. Experimental setup for a CCD TID radiation test is shown in Figure 1.

The sample used in the experiment is DALSA's IL-P3-B linear CCD. The linear CCD includes 2048 pixels and exhibits high resolution. The pixel size is $14 \times 14 \mu \mathrm{m}^{2}$. The IL-P3$\mathrm{B}$ linear CCD has a data rate of $40 \mathrm{MHz}$. Block diagram of DALSA's IL-P3-B linear CCD is shown in Figure 2 [7].

\section{Results and Discussion}

3.1. Increase of Dark Current. The dark current is the current measured in the absence of incident photons, which is expressed in $\mathrm{e} / \mathrm{s} /$ pixel or $\mathrm{nA} / \mathrm{cm}^{2}$. The dark current in a CCD is the current which is not generated by photoelectric effects. As the European Machine Vision Association 1288 (EMVA1288) standard, the dark current is given as [8]

$$
\mu_{d}=\mu_{d 0}+\mu_{I} t_{\text {exp }}
$$

where $\mu_{I}$ is the dark current, $\mu_{d 0}$ is the average number of electrons without light for exposure time zero, and $\mu_{d}$ is the average number of electrons without light.

Both TID and displacement radiation induce the increase of dark current. It is known that the increase of dark current in a CCD is mainly caused by TID radiation damage, so we will focus on the TID radiation-induced increase of dark current.

There are several dark current sources such as thermal generation, surface generation, tunneling, and impact ionization. The main sources of the increase of dark current are thermal generation at the space charge region (SCR) and thermal generation caused by surface states at the $\mathrm{Si}$ and $\mathrm{SiO}_{2}$ interface induced by TID radiation.

Ionizing radiation creates new interface states between the gate oxide and bulk silicon. Since the energy level of the newly formed states lies within band-gap, their presence typically causes the dark current generation increase [9]. The dark current versus the TID is shown in Figure 3. When the TID is lower than $10 \mathrm{krad}(\mathrm{Si})$, the dark current increases slightly. However, when the TID is higher than $10 \mathrm{krad}(\mathrm{Si})$, the dark current increases remarkably. The reason is that the ionizing radiation damage is worse with the accumulation of TID. Ionizing radiation causes the interface state increase which induces the increase of dark current generation rate.

Ionizing damage causes an increase in the density of oxide traps and interface traps of CCDs. The generation of the traps will increase the electron-hole pair generation, which increases the dark current [6]. The creation of traps will increase the width of SCR by changing the potential of the $\mathrm{Si} / \mathrm{SiO}_{2}$ interface, which will induce the increase of dark current. As Shockley-Read-Hall theory, the generation rate, $G$, is expressed as [5]

$$
G=\frac{\sigma_{n} \sigma_{p} v_{\mathrm{th}} D_{t}\left(n_{i}^{2}-p n\right)}{\sigma_{n}\left[n+n_{i} \exp \left\{\left(E_{t}-E_{i}\right) / k T\right\}\right]+\sigma_{p}\left[p+n_{i} \exp \left\{-\left(E_{t}-E_{i}\right) / k T\right\}\right]},
$$

where $\sigma_{n}$ and $\sigma_{p}$ represent the captured cross sections of electrons and holes, $v_{\text {th }}$ is the thermal velocity, and $D_{t}$ represents the trap concentration.

For the depleted surface $n \ll n_{i}$ and $p \ll n_{i}$,

$$
G=\frac{\sigma_{n} \sigma_{p} v_{\mathrm{th}} D_{t}\left(n_{i}^{2}-p n\right)}{\sigma_{n}\left[n+n_{i} \exp \left\{\left(E_{t}-E_{i}\right) / k T\right\}\right]+\sigma_{p}\left[p+n_{i} \exp \left\{-\left(E_{t}-E_{i}\right) / k T\right\}\right]} .
$$




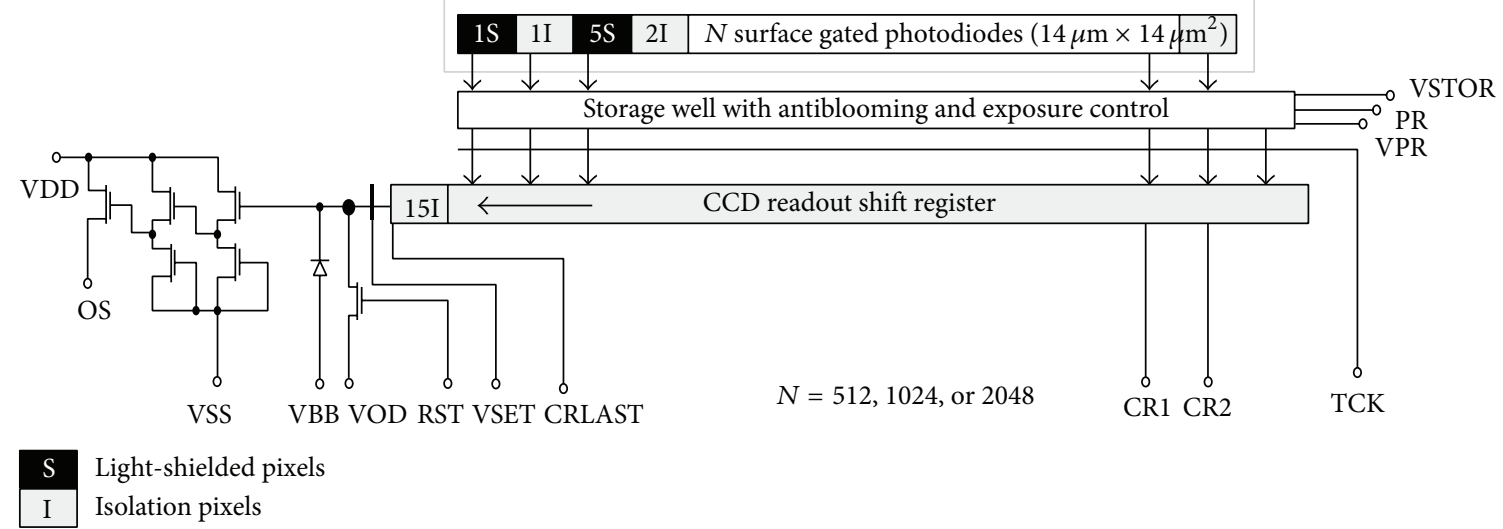

FIGURE 2: Block diagram of DALSA's IL-P3-B linear CCD [7].

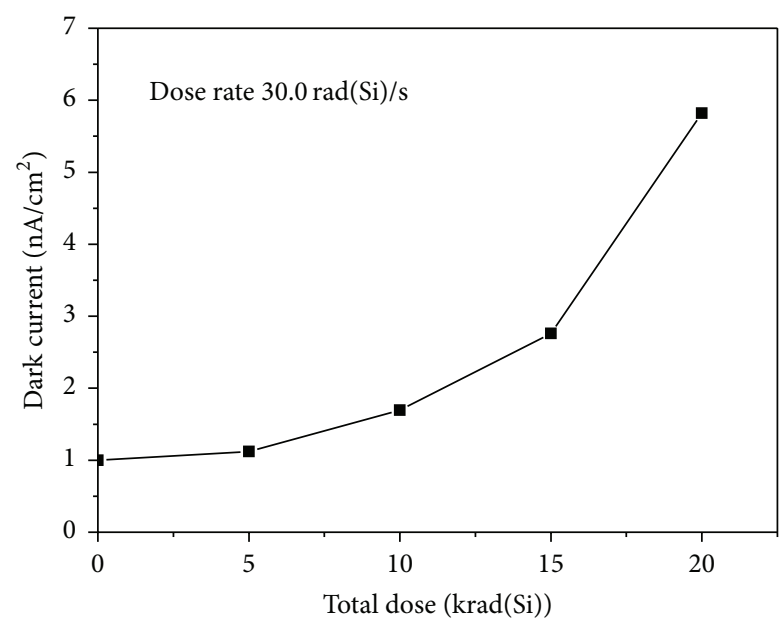

FIgURE 3: Dark current versus the TID at $30.0 \mathrm{rad}(\mathrm{Si}) / \mathrm{s}$.

The dark current, $\mu_{I}$, is expressed as

$$
\begin{aligned}
& \mu_{I}=\int_{E_{V}}^{E_{C}} G d E_{t}, \\
& \mu_{I}=\frac{\pi}{2}\left(\sigma_{n} \sigma_{p}\right)^{1 / 2} v_{\mathrm{th}} k D_{t} T n_{i} .
\end{aligned}
$$

3.2. Increase of DSNU and PRNU. The CCD readout images include a series of pixel output and vary from pixel to pixel. The inhomogeneities are of no noise which makes the output signal vary in time. The inhomogeneities may only distribute randomly, so it is better to name this effect nonuniformity [5]. Essentially, there are two basic nonuniformities. First, the dark signal is various in different pixels. This effect is called DSNU. Second, the variation of the sensitivity is called PRNU. Both the DSNU and the PRNU of a CCD will be degraded by TID damage.
The DSNU represents the root mean square deviation of all effective pixel outputs of the imaging area in dark environments. As the EMVA1288 standard, the DSNU is given by [8]

$$
\begin{aligned}
& \mu_{y, \text { dark }}=\frac{1}{M N} \sum_{m=0}^{M-1} \sum_{n=0}^{N-1} y_{\text {dark }}[m][n], \\
& s_{y, \text { dark }}^{2}=\frac{1}{M N-1} \sum_{m=0}^{M-1} \sum_{n=0}^{N-1}\left(y_{\text {dark }}[m][n]-\mu_{y, \text { dark }}\right)^{2}, \\
& \text { DSNU }=s_{y, \text { dark }},
\end{aligned}
$$

where $M$ and $N$ are the number of rows and columns of the image, $m$ and $n$ are the row and column indices of the array, $y_{\text {dark }}[m][n]$ is the mean dark signal of all $[m][n]$ pixels in all the captured dark images, $\mu_{y \text {,dark }}$ is the mean dark signal of all the captured dark images, and $s_{y \text {,dark }}^{2}$ is the spatial variance of all the captured dark images.

Figure 4 shows the DSNU increases with increasing TID. When the TID is higher than $10 \mathrm{krad}(\mathrm{Si})$, the DSNU increases remarkably. This is because the TID damage is worse with the accumulation of TID. TID radiation induces an increase not only in the dark signal but also in DSNU. The DSNU is due to the dark signal fluctuations within pixels of the CCDs induced by a fluctuation in the number of generation states. The fluctuation increases with increasing TID. When the TID is lower than $10 \mathrm{krad}(\mathrm{Si})$, the number of generation states is small and has a limited influence on the DSNU.

The PRNU is the nonuniformity of the sensitivity of photo response. The PRNU is defined as a standard deviation relative to the mean value. The PRNU gives the spatial standard deviation of the photo response nonuniformity in \% from the mean. As the EMVA1288 standard, the PRNU is given by [8]

$$
\mu_{y, 50}=\frac{1}{M N} \sum_{m=0}^{M-1} \sum_{n=0}^{N-1} y_{50}[m][n]
$$




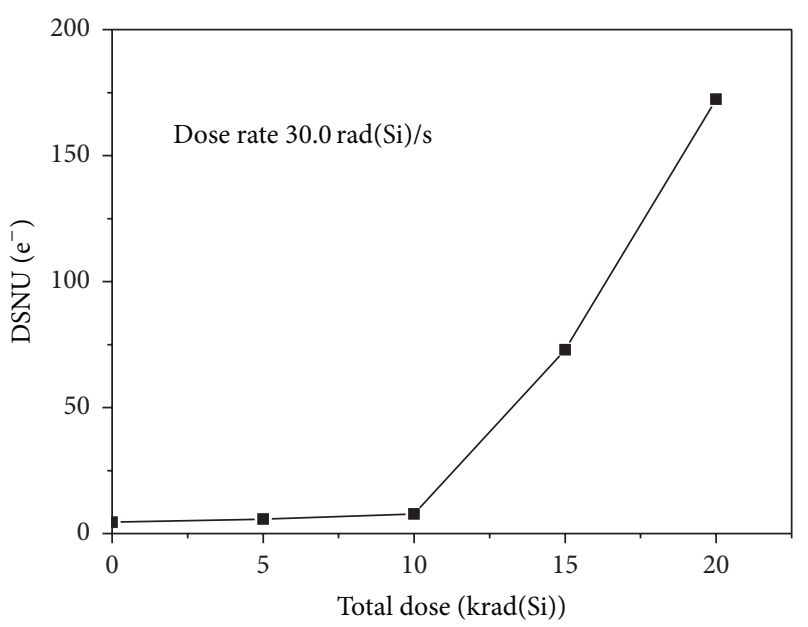

FIgURE 4: DSNU versus the TID at $30.0 \mathrm{rad}(\mathrm{Si}) / \mathrm{s}$.

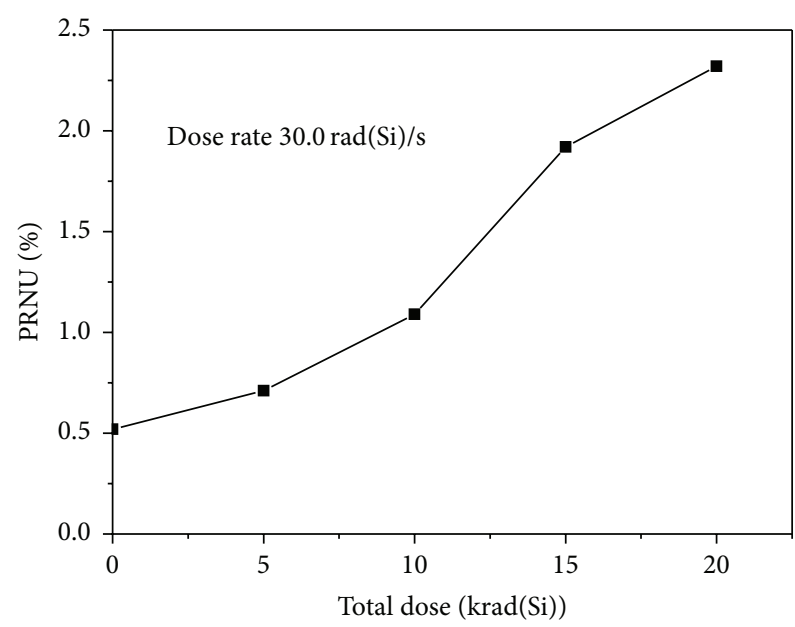

FIGURE 5: PRNU versus the TID at $30.0 \mathrm{rad}(\mathrm{Si}) / \mathrm{s}$.

$$
\begin{aligned}
s_{y, 50}^{2} & =\frac{1}{M N-1} \sum_{m=0}^{M-1} \sum_{n=0}^{N-1}\left(y_{50}[m][n]-\mu_{y, 50}\right)^{2}, \\
\text { PRNU } & =\frac{\sqrt{s_{y, 50}^{2}-s_{y, \text { dark }}^{2}}}{\mu_{y, 50}-\mu_{y, \text { dark }}} \times 100 \%,
\end{aligned}
$$

where $M$ and $N$ are the number of rows and columns of the image, $m$ and $n$ are the row and column indices of the array, $y_{50}[m][n]$ is the mean of all $[m][n]$ pixels in the $50 \%$ saturation images, $\mu_{y, 50}$ is the mean of the $50 \%$ saturation images, and $s_{y, 50}^{2}$ is the spatial variance of $50 \%$ saturation images.

Figure 5 shows the PRNU increases with increasing TID. The PRNU increase is caused by the oxide traps and the interface traps induced by TID radiation. These traps can capture the signal charges in the potential well and then emit them, which increases the signal charge fluctuations within pixels of the CCDs. The fluctuation increases with increasing TID, so the PRNU also increases with increasing TID.

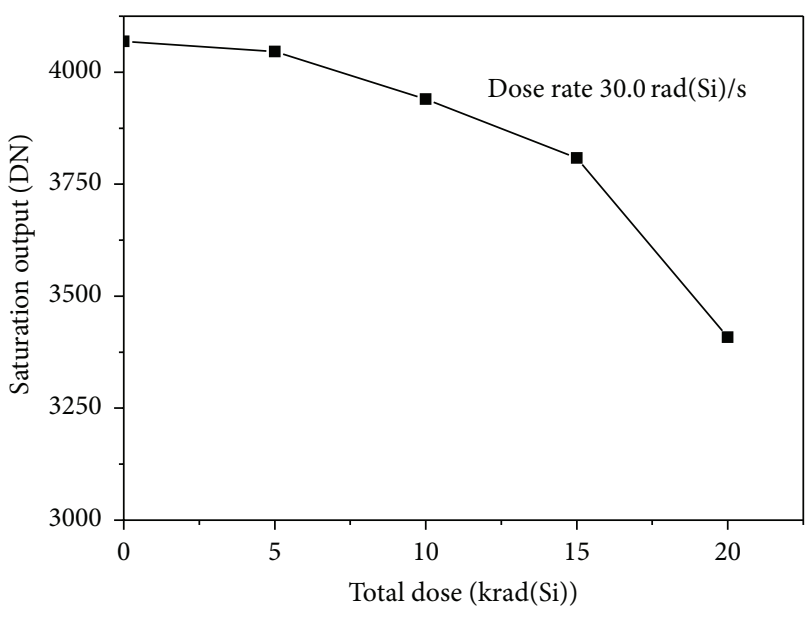

FIGURE 6: Saturation output versus the TID at $30.0 \mathrm{rad}(\mathrm{Si}) / \mathrm{s}$.

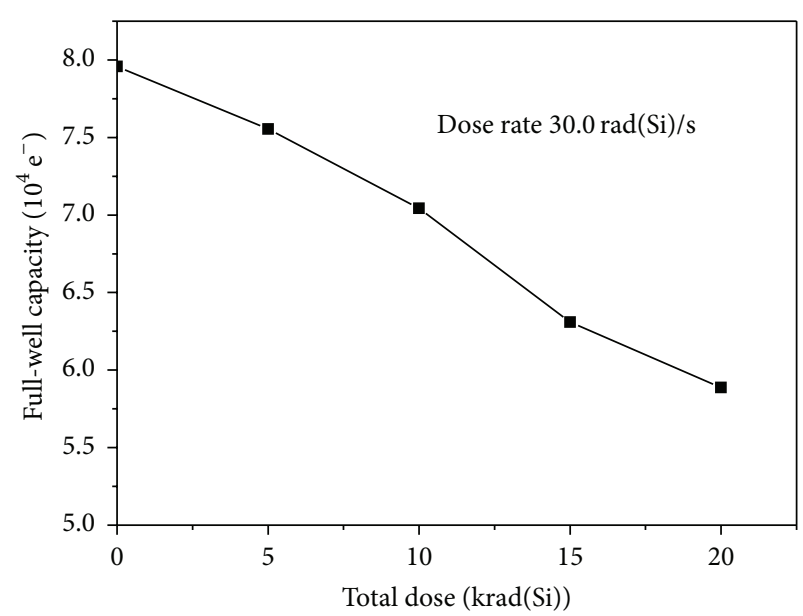

Figure 7: Full-well capacity versus the TID at $30.0 \mathrm{rad}(\mathrm{Si}) / \mathrm{s}$.

3.3. Decrease of Saturation Output and FWC. The saturation output is the maximum output signal with all pixels illuminated to saturation. The saturation output as a function of $\mathrm{TID}$ at $30.0 \mathrm{rad}(\mathrm{Si}) / \mathrm{s}$ is shown in Figure 6. From Figure 6, one can see that the saturation output decreases with increasing TID. When the TID is higher than $10 \mathrm{krad}(\mathrm{Si})$, the saturation output decreases remarkably. The degradation of the saturation output is due to the depth of the potential wells in the shift register region and the imaging region decrease induced by TID damage.

It has been known that the TID damage induces the threshold voltage shift of CCD readout shift register as shown in Figure 2. The threshold voltage shift causes a decrease of the potential well depth. So the saturation output filled in the full potential well also decreases.

The FWC is calculated by dividing the saturation output (DN) by conversion factor (DN/e ${ }^{-}$). The FWC versus the TID at $30.0 \mathrm{rad}(\mathrm{Si}) / \mathrm{s}$ is shown in Figure 7. From Figure 7, one can see that FWC decreases with increasing TID. This is due to the decrease of saturation output and the increase of conversion 


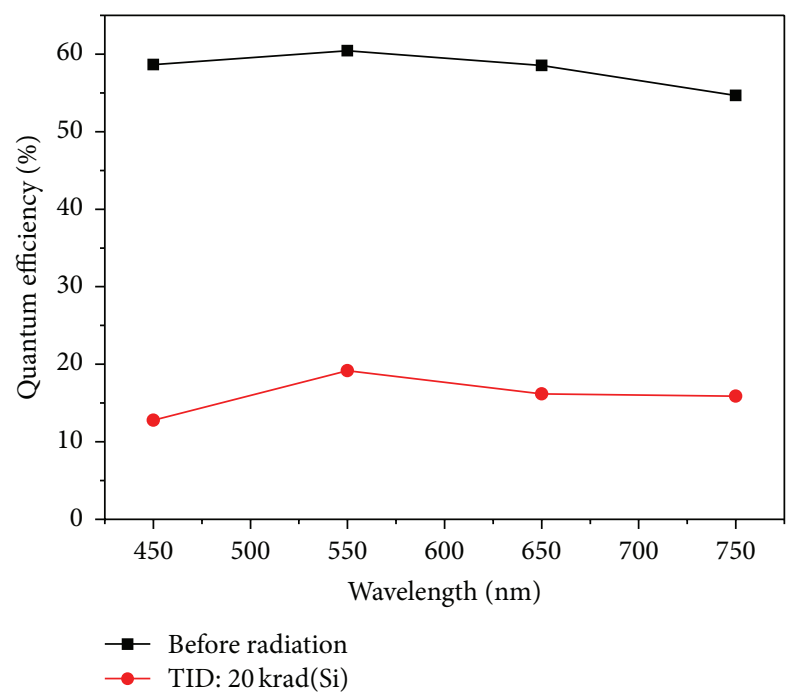

FIGURE 8: QE versus the wavelength before and after $20 \mathrm{krad}(\mathrm{Si})$ radiation.

factor. The experiment results show that the conversion factor increases a little after TID radiation.

3.4. Decrease of $\mathrm{QE}$ and Responsivity. $\mathrm{QE}$ and spectral responsivity represent how a CCD responds to the impinged photons. TID damage will cause a decrease of $\mathrm{QE}$ and spectral responsivity in a CCD. QE is a quantitative parameter that reflects the photon-sensitivity of an image sensor as a function of the wavelength (i.e., the energy) of impinging photons. It is given by [10]

$$
\mathrm{QE}=\frac{N_{\mathrm{sig}}(\lambda)}{N_{\mathrm{ph}}(\lambda)},
$$

where $N_{\text {sig }}$ is the collected video signal charge, $N_{\text {ph }}$ is the number of injected photons, and $\lambda$ stands for the wavelength.

The QE loss is mainly due to two limitations. The first is the impinging loss which represents the photon loss during the impinging procedures. It includes the loss from the optical system and the absorption and reflection by the structures above the photodiode (e.g., the metal and dielectric layers). In other words, the impinging loss stands for the missing photons that do not make it to the surface of the photosensing region. Secondly, the collection of the photon-generated carriers is not one hundred percent efficient, which thus introduces a QE reduction [10].

The QE versus the wavelength before and after $20 \mathrm{krad}(\mathrm{Si})$ radiation at $30.0 \mathrm{rad}(\mathrm{Si}) / \mathrm{s}$ is shown in Figure 8. The QE decreases remarkably after $20 \mathrm{krad}(\mathrm{Si})$ radiation. The photongenerated carriers within the depletion region can be collected without any loss because of the existence of the buildin electrical field. However, the carriers generated outside the depletion region may be recombined before diffusing to the depletion region. The threshold voltage shift induced by TID damage causes the deep depletion region decrease, and thus the collected photon-generated carriers decrease. The increase in density of traps induced by TID damage causes

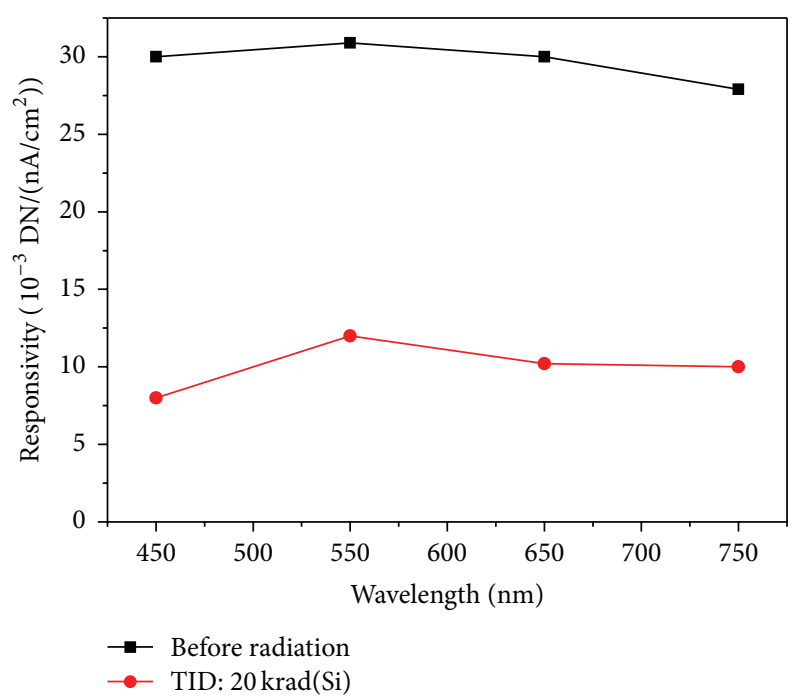

FIGURE 9: Responsivity versus the wavelength before and after $20 \mathrm{krad}(\mathrm{Si})$ radiation.

the impinging loss of photon-generated carriers. So the $\mathrm{QE}$ decreases remarkably after $20 \mathrm{krad}(\mathrm{Si})$ radiation.

The responsivity is also used to characterize the photonsensitivity of a CCD. It is the ratio of photocurrent to optical input power and is given by [10]

$$
R=\frac{I_{\mathrm{ph}}}{P}=\mathrm{QE} \frac{q \lambda}{h c},
$$

where $I_{\mathrm{ph}}$ is the photocurrent and $P$ is the optical input power.

The responsivity versus the wavelength before and after $20 \mathrm{krad}(\mathrm{Si})$ radiation at $30.0 \mathrm{rad}(\mathrm{Si}) / \mathrm{s}$ is shown in Figure 9. The responsivity also decreases remarkably after $20 \mathrm{krad}(\mathrm{Si})$ radiation. From (8), we can conclude that the responsivity degradation mechanisms are similar to the QE.

\section{Conclusion}

The radiation effects on the DALSA CCD at $30.0 \mathrm{rad}(\mathrm{Si}) / \mathrm{s}$ were investigated. The behavior of the tested CCD had shown a remarkable degradation after ${ }^{60} \mathrm{Co} \gamma$-ray radiation. The dark current increases with increasing TID, which is due to the increase in density of traps induced by TID damage. The DSNU and PRNU increase with increasing TID, which is due to the increase of the signal charge fluctuations (both dark and illumination environments) within pixels of a CCD induced by TID damage. When the TID is higher than $10 \mathrm{krad}(\mathrm{Si})$, the dark current, DSNU, and PRNU increase remarkably. This means that the CCD used in the radiation environments where the TID is higher than $10 \mathrm{krad}(\mathrm{Si})$ should consider the serious degradation of the performance.

The saturation output and FWC decrease with increasing TID, which is mainly due to the decrease of the potential well depth in the CCD induced by TID damage. When the TID is higher than $10 \mathrm{krad}(\mathrm{Si})$, the saturation output and FWC decrease remarkably. The QE and responsivity decrease with increasing TID, which is due to the deep depletion region 
decrease and the impinging loss of photon-generated carriers induced by TID damage. When the TID is $20 \mathrm{krad}(\mathrm{Si})$, the $\mathrm{QE}$ and responsivity decrease remarkably, which induces the remarkable decrease of the photon-sensitivity of a CCD.

In order to study dose rate effects on the CCDs, more radiation experiments and annealing tests on the CCDs will be carried out in the laboratory to compare the degradations under the same total ionizing doses at $0.03,0.3$, and $3.0 \mathrm{rad}(\mathrm{Si}) / \mathrm{s}$. More detailed experimental results will be provided.

\section{Competing Interests}

The authors declare that they have no competing interests.

\section{Acknowledgments}

This work was supported by the National Science Foundation of China (Grants nos. 11305126, 11235008) and the Foundation of State Key Laboratory of China (Grant no. SKLIPR1211).

\section{References}

[1] K. Abe, A. Arodzero, C. Baltay et al., "Design and performance of the SLD vertex detector: a 307 Mpixel tracking system," Nuclear Instruments and Methods in Physics Research Section A: Accelerators, Spectrometers, Detectors and Associated Equipment, vol. 400, no. 2-3, pp. 287-343, 1997.

[2] A. Yamashita, T. Dotani, H. Ezuka, M. Kawasaki, and K. Takahashi, "Performance of the X-ray CCDs aboard the ASCA satellite after 5-year operation in space," Nuclear Instruments and Methods in Physics Research A, vol. 436, no. 1-2, pp. 68-73, 1999.

[3] T. Hardy, R. Murowinski, and M. J. Deen, "Charge transfer efficiency in proton damaged CCD's," IEEE Transactions on Nuclear Science, vol. 45, no. 2, pp. 154-163, 1998.

[4] A. Simone, I. Debusschere, A. Alaerts, and C. Claeys, "Ionizing radiation hardening of a CCD technology," IEEE Transactions on Nuclear Science, vol. 39, no. 6, pp. 1964-1973, 1992.

[5] G. R. Hopkinson, "Radiation-induced dark current increases in CCDs," in Proceedings of the 2nd European Conference on Radiation and its Effects on Components and Systems (RADECS '93), pp. 401-408, St. Malo, France, September 1993.

[6] Z. Wang, W. Chen, S. Huang, M. Liu, B. He, and J. Sheng, "Degradation of saturation output of the COTS array chargecoupled devices induced by total dose radiation damage," Nuclear Instruments and Methods in Physics Research Section A: Accelerators, Spectrometers, Detectors and Associated Equipment, vol. 751, pp. 31-35, 2014.

[7] Dalsa, Dalsa IL-P3-B image sensors datasheet, 2003.

[8] European Machine Vision Association (EMVA), "Standard for characterization of image sensors and cameras," EMVA Standard 1288, 2010.

[9] J. R. Janesick, T. Elliott, and F. Pool, "Radiation damage in scientific charge-coupled devices," IEEE Transactions on Nuclear Science, vol. 36, no. 1, pp. 572-578, 1989.

[10] X. Wang, Noise in sub-micron CMOS image sensors [Ph.D. dissertation], Delft University of Technology, Delft, The Netherlands, 2008. 


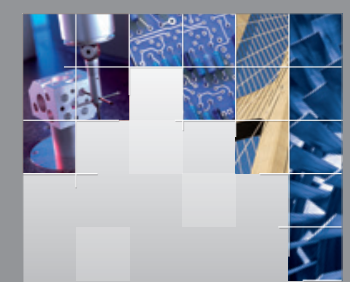

\section{Enfincering}
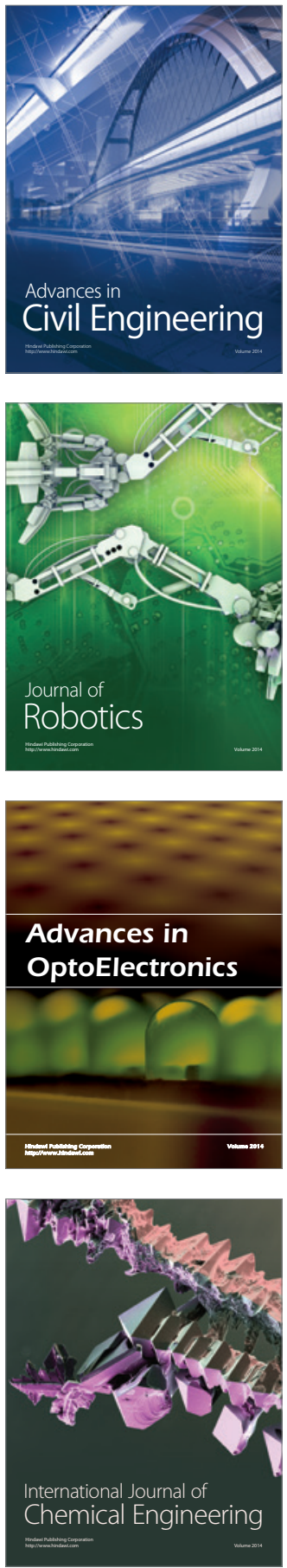

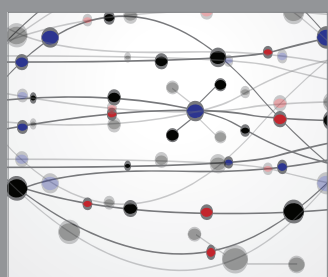

The Scientific World Journal

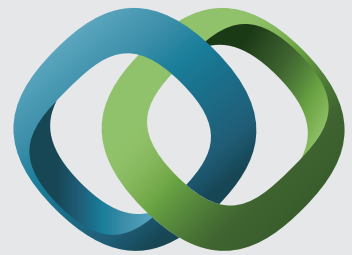

\section{Hindawi}

Submit your manuscripts at

http://www.hindawi.com
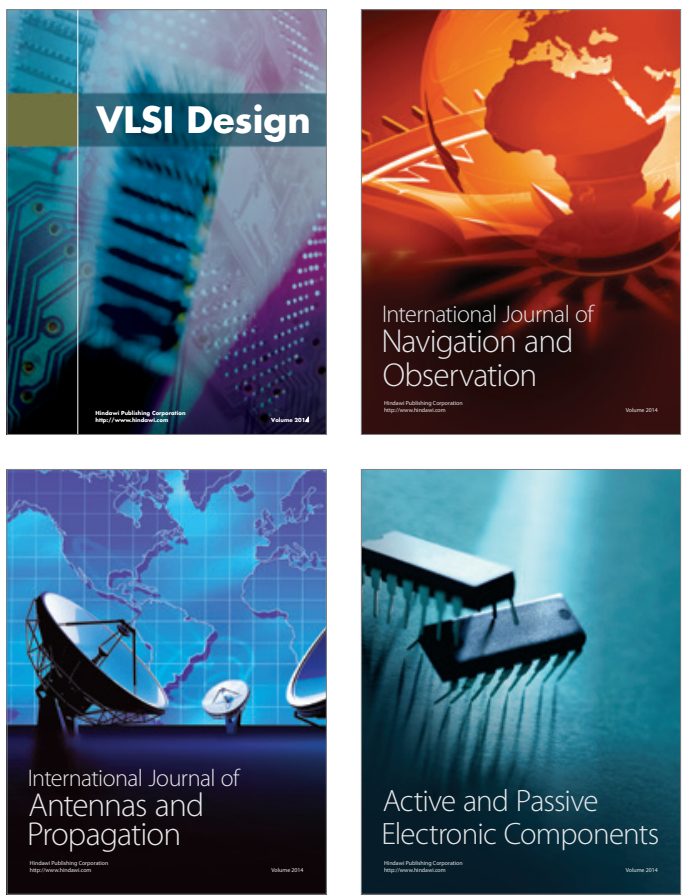
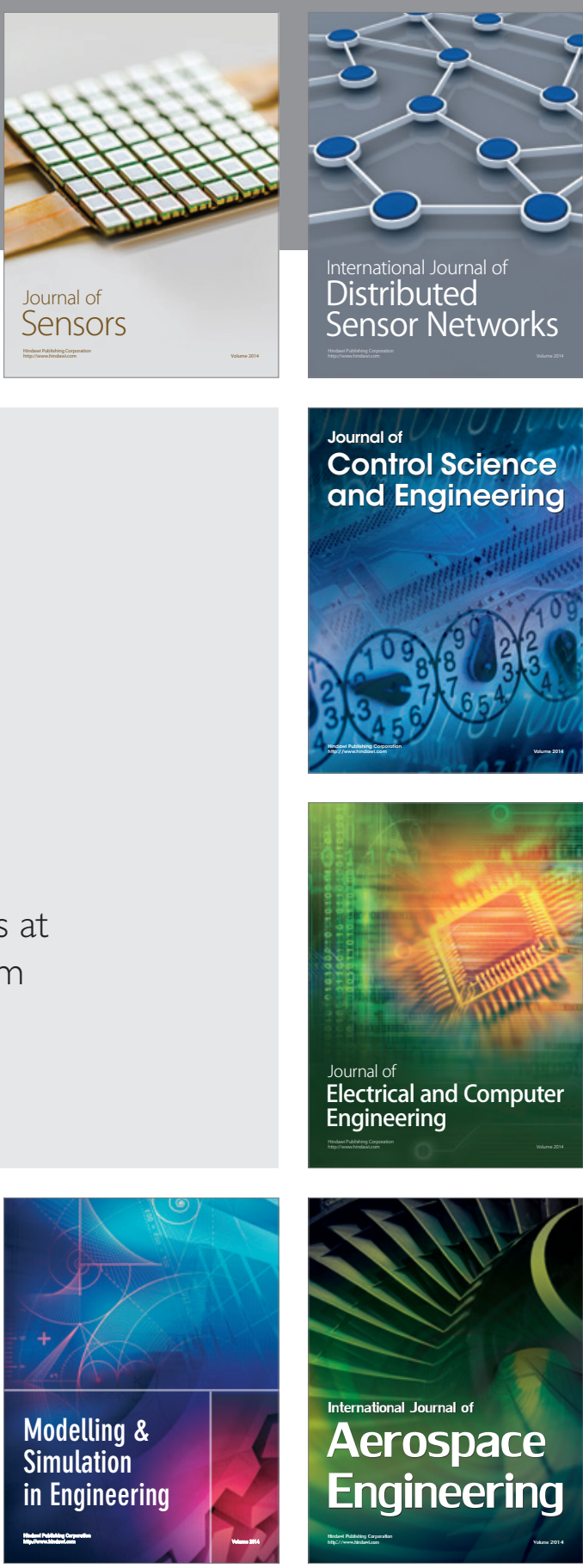

International Journal of

Distributed

Sensor Networks

Journal of

Control Science

and Engineering
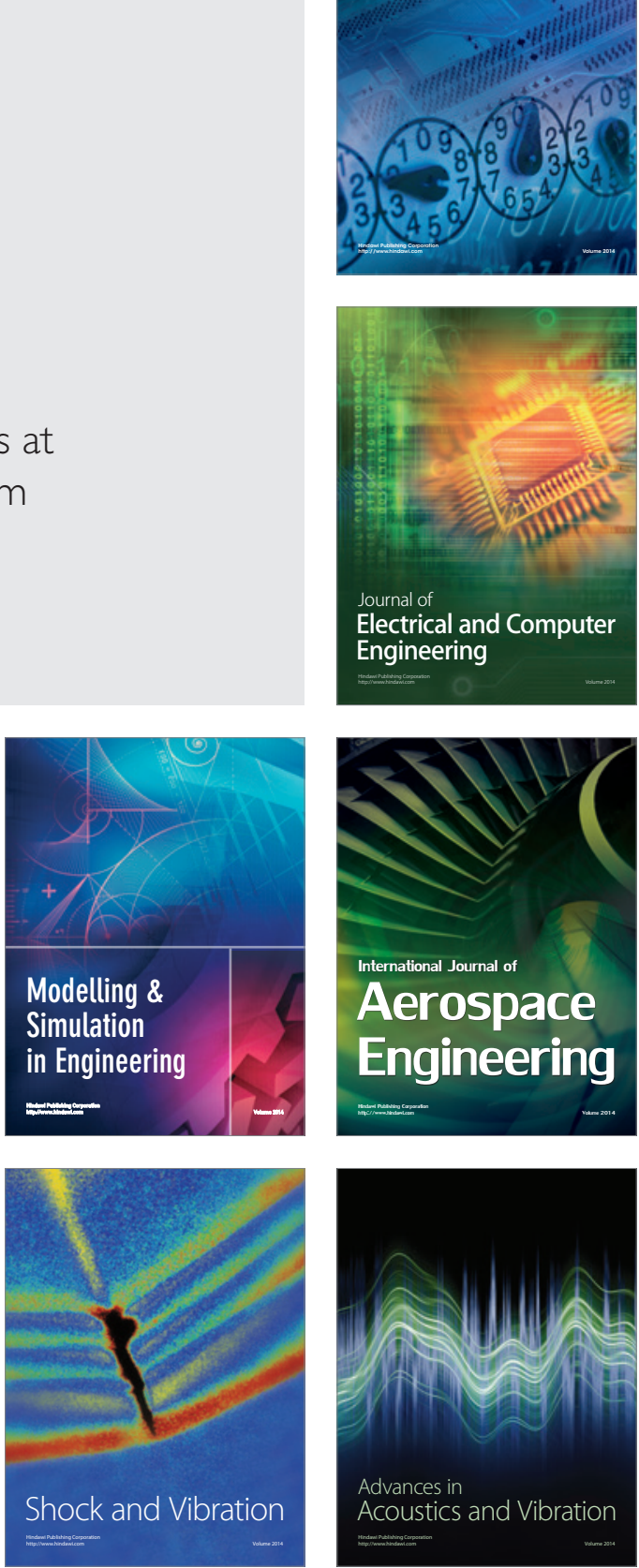